\title{
Pirh2 interacts with and ubiquitylates signal recognition particle receptor $\beta$ subunit
}

\author{
Kenji Abe, Takayuki Hattori, Tomoyasu Isobe, Kyoko Kitagawa, Toshiaki Oda, Chiharu Uchida and \\ Masatoshi Kitagawa \\ Department of Biochemistry 1, Hamamatsu University School of Medicine, 1-20-1 Handayama, Higashi-ku, Hamamatsu, Shizuoka \\ 431-3192, Japan
}

(Received 26 November 2007; and accepted 7 December 2007)

\begin{abstract}
Pirh2 is a RING finger type ubiquitin ligase which ubiquitylates various proteins including p53, $\mathrm{p} 27^{K i p l}, \mathrm{HDAC} 1$, and $\varepsilon$-COP. In this study, we identified signal recognition particle receptor $\beta$ subunit (SR $\beta$ ), an integral membrane protein of the endoplasmic reticulum (ER), as a novel Pirh2-interacting protein by yeast two-hybrid screening. We confirmed that Pirh2 interacted with SR $\beta$ in mammalian cells. An immunofluorescent staining revealed that Pirh2 colocalized with SR $\beta$ in the ER. Pirh2 poly-ubiquitylated SR $\beta$ in an intact RING finger domain-dependent manner in vivo and in vitro. Unexpectedly, different from other Pirh2 substrates, neither overexpression of Pirh2 nor depletion of cellular Pirh2 affected SR $\beta$ protein stability. Pirh2 preferentially utilized lysine residues 6 and 29 of the ubiquitin to mediate the formation of polyubiquitin chains on SR $\beta$. These results suggest that Pirh2 may regulate SR $\beta$ function by mediating poly-ubiquitylation of SR $\beta$ without affecting the stability of SR $\beta$ protein per se.
\end{abstract}

Ubiquitylation is a versatile post-translational modification mechanism used by eukaryotic cells (10). It is catalyzed through a pathway involving three enzymes, E1 (ubiquitin-activating enzyme), E2 (ubiquitin-conjugating enzyme), and E3 (ubiquitin ligase). E3 is the most diverse, and it determines substrate specificity and the rate of ubiquitin conjugation. Substrates can be modified with monoubiquitin or with a polyubiquitin chain that is linked through lysines present within ubiquitin itself. The most common polyubiquitylation is linked through lysine 48 of ubiquitin which serves as a signal for the rapid degradation of substrate by the proteasome-dependent pathway (2). However, recent studies have

Address correspondence to: Masatoshi Kitagawa, Ph.D., Department of Biochemistry 1, Hamamatsu University School of Medicine, 1-20-1 Handayama, Higashi-ku, Hamamatsu, Shizuoka 431-3192, Japan

Tel: +81-53-435-2322, Fax: +81-53-435-2322

E-mail: kitamasa@hama-med.ac.jp revealed roles other than proteolysis for polyubiquitylation (17). While lysine 48- and lysine 29-linked polyubiquitin chains mediate proteasome-dependent degradation, lysine 63-linked polyubiquitin chains are a signal for endocytosis, IкB kinase activation, ribosome modification, and DNA repair $(4,7,11$, $20,21)$. Although it has been reported that lysine 6 -linked polyubiquitin chains are deubiquitylated by the $26 \mathrm{~S}$ proteasome and are not thought to be a proteasomal degradation signal (15), detailed biological function of lysine 6-linked polyubiquitin is not well understood.

A RING-H2 type E3 ubiquitin ligase Pirh2 (p53inducible protein with RING-H2 domain), also known as androgen receptor $\mathrm{N}$-terminal-interacting protein (ARNIP) (1), was identified as a p53-inducible and -interacting protein which promotes ubiquitylation and degradation of p53 (12). Other studies have shown that Pirh2 interacts with various molecules including histone acetylase TIP60 (Tatinteractive protein of $60 \mathrm{kDa}$ ) (18), measles virus 
phosphoprotein (3), N-terminal kinase-like proteinbinding protein 1 (NTKL-BP1) (22), and calmodulin (5). Recently, it has been reported that histone deacetylase 1 (HDAC1) and the $\varepsilon$-subunit of coatomer complex ( $\varepsilon$-COP) were ubiquitylated and subsequently degraded by Pirh2 $(13,14)$. Moreover, we had found that Pirh2 promotes ubiquitin-dependent degradation of the CDK inhibitor p $27^{\text {Kip } 1}$ at the G1/S phase of the cell cycle (9). These studies suggest that Pirh2 is involved in various cellular events by ubiquitylating and/or interacting with these molecules.

Secretory proteins are synthesized with an N-terminal hydrophobic signal sequence. As the signal sequence emerges from the ribosome, it is recognized by the signal recognition particle (SRP) and subsequently targeting of the ribosome-nascent chain complex to the endoplasmic reticulum (ER) membrane occurs. At the membrane, SRP contacts the SRP receptor (SR), a heterodimer composed of SR $\alpha$ and SR $\beta$. Contact between SRP and SR $\alpha$ leads to the transfer of the nascent chain from SRP into the lumen of the ER through the translocation channel formed by the Sec61p complex $(6,8)$.

In this study, we identified SR $\beta$ as a novel Pirh2interacting protein by yeast two-hybrid screening. We found that Pirh2 promoted ubiquitylation of SR $\beta$ without affecting the stability of SR $\beta$. Our findings suggest that Pirh2 may control the synthesis of secretory protein by the functional regulation of SR $\beta$ via ubiquitylation.

\section{MATERIALS AND METHODS}

Yeast two-hybrid screening. The yeast two-hybrid screening was performed with the Matchmaker TwoHybrid System 2 (Clontech, Mountain View, CA, USA). The full-length rat Pirh2 was cloned into the yeast two-hybrid vector pAS2-1 and the plasmid was transformed into the yeast followed by screening a pACT2 rat brain cDNA library (Clontech) according to the manufacturer's instructions.

Cell culture, reagents, and antibodies. All cells were maintained in Dulbecco's modified Eagle's medium supplemented with $10 \%$ heat-inactivated fetal bovine serum and antibiotics. MG132 (Peptide institute, Osaka, Japan) and cycloheximide (Wako, Osaka, Japan) were purchased. Polyclonal antibody against Pirh2 (Calbiochem, San Diego, CA, USA) was purchased. Monoclonal antibodies against FLAG-epitope (M2; Sigma, St. Louis, MO, USA), HA-epitope (12CA5; Roche, Basel, Switzerland), Xpress-epitope
(Invitrogen, Carlsbad, CA, USA), $\alpha$-tubulin (DM1A; Sigma), and $\beta$-actin (AC-15; Sigma) were used for immunoprecipitation and/or immunoblotting. Polyclonal antibody against SR $\beta$ was raised in rabbit by standard procedures using the carboxy-terminus fragment of recombinant GST-hSR $\beta$ (residues 87-271) as antigens and purified by a Melon ${ }^{\mathrm{TM}} \mathrm{Gel}$ IgG Purification kit (PIERCE, Rockford, IL, USA).

Mammalian expression vectors and transfection. Human wild-type and RING finger mutant (C148A) Pirh2 expression vectors were constructed as described previously (9). Human SR $\beta$ cDNA was amplified by RT-PCR from HCT116 cell RNA and cloned into the pcDNA4-HisMax expression vector (Invitrogen). The resultant pcDNA4-HisMax-SR $\beta$ encodes $6 \times$ His and the Xpress epitope tag fused with the amino terminus of human SR $\beta$. pCMV-2× FLAG-SR $\beta$ was constructed by ligating FLAG-SR $\beta$ cDNA into the pCMV-Tag2 expression vector (Stratagene, La Jolla, CA, USA). HEK293 and COS-1 cells were transfected by the calcium phosphate method. U2OS cells were transfected using FuGENE6 (Roche) according to the manufacturer's protocol. Small interfering RNA (siRNA) duplexes for Pirh2 were described previously (9). Non-silencing control siRNA was obtained from Qiagen (Valencia, CA, USA). The cells were transfected with siRNA by using Oligofectamine (Invitrogen) according to manufacturer's protocol.

Immunoprecipitation and immunoblotting. Cells were lysed in immunoprecipitation lysis buffer $(0.5 \%$ Triton X-100, $150 \mathrm{mM} \mathrm{NaCl}, 20 \mathrm{mM}$ Tris-HCl, $\mathrm{pH}$ 7.6, and protease inhibitor mix). Immunoprecipitation and immunoblotting were performed as described previously (9).

Immunofluorescence. COS-1 cells were cotransfected with Xpress-Pirh 2 and $2 \times$ FLAG-SR $\beta$ expression plasmids. After $48 \mathrm{~h}$, the cells were fixed with $4 \%$ paraformaldehyde in PBS $(-)$, permeabilized with $0.2 \%$ Triton X-100 in PBS $(-)$, blocked with $10 \%$ normal goat serum, and treated with anti-FLAG antibody conjugated with AlexaFluor546 and antiXpress antibody conjugated with AlexaFluor488. The ER was stained using the ER labeling kit according to the manufacturer's protocol (Molecular Probe, Eugene, OR, USA). Fluorescence images were taken under fluorescence microscope system (Biozero, KEYENCE, Tokyo, Japan). 
In vitro ubiquitylation assay. GST-tagged FLAGSR $\beta$, Pirh2, and Pirh2 (C148A) proteins were expressed in Escherichia Coli BL21-CodonPlus (DE3) (Stratagene) and affinity-purified with glutathioneSepharose 4B (GE Healthcare, Buckinghamshire, UK), and then the GST tag was removed by cleavage with PreScission protease (GE Healthcare). FLAG-SR $\beta$ protein was incubated with or without Pirh2 protein at $30^{\circ} \mathrm{C}$ for $60 \mathrm{~min}$ in a $20 \mu \mathrm{L}$ ubiquitylation mixture supplemented with $50 \mathrm{mM}$ Tris$\mathrm{HCl}, \mathrm{pH} 8.3,5 \mathrm{mM} \mathrm{MgCl}_{2}, 2 \mathrm{mM}$ DTT, $10 \mathrm{mM}$ phosphocreatinine, 0.2 units $/ \mathrm{mL}$ phosphocreatinine kinase, $5 \mathrm{mM}$ adenosine-5'-triphosphate, $2 \mu \mathrm{L}$ of GST-ubiquitin or His-ubiquitin, $250 \mu \mathrm{M}$ MG132, protease inhibitor mix, E1 (120 ng, Boston Biochem, Cambridge, MA, USA), and UbcH5b (500 ng, Boston Biochem). After incubation, the reactants were subjected to immunoblotting with anti-FLAG antibody.

\section{RESULTS}

\section{Identification of SR $\beta$ as a Pirh2-interacting protein}

To identify novel interacting proteins with Pirh2, we performed yeast two-hybrid screening of a rat brain cDNA library using full-length Pirh2 as bait and found that Pirh2 interacted with signal recognition particle receptor $\beta$ subunit (SR $\beta$ ), a membrane protein of the endoplasmic reticulum (ER) essential for protein translocation across the ER membrane. To determine whether SR $\beta$ physically interacts with Pirh2 in mammalian cells, we coexpressed the $4 \times$ FLAG-Pirh2 and Xpress-SR $\beta$ in HEK293 cells. When extracts from transfected cells were immunoprecipitated with anti-FLAG antibody and immunoblotted with anti-Xpress antibody, SR $\beta$ was only coprecipitated in the presence of transfected Pirh2 (Fig. 1A, IP: FLAG and IB: Xpress). In a reciprocal experiment using anti-Xpress antibody for immunoprecipitation, coprecipitated Pirh2 was observed in cells transfected with the Xpress SR $\beta$ plasmid but not in cells transfected with an empty vector (Fig. 1A, IP: Xpress and IB: FLAG). To further confirm this interaction, we examined whether transfected SR $\beta$ would associate with endogenous Pirh2. HEK293 cells were transfected with $2 \times$ FLAG SR $\beta$, and the cell extracts were subjected to immunoprecipitation with anti-FLAG antibody and immunoblotted with anti-Pirh2 antibody. As shown in Fig. 1B, endogenous Pirh2 was coprecipitated with SR $\beta$ only when $2 \times$ FLAG SR $\beta$ was transfected. Taken together, these results suggest that Pirh2 and SR $\beta$ physically interact with each other in mammalian cells. In order to examine whether Pirh2 colocalizes with $\operatorname{SR} \beta$ in cells, we transfected $2 \times$ FLAG-SR $\beta$ with or without Xpress-Pirh2 into COS-1 cells and stained them immunocytochemically with fluorescent-conjugated antibodies. In this experiment, SR $\beta$ (Figs. 1C-E), Pirh2 (Figs. 1F-H), and ER (Figs. 1I-K) were stained red, green, and blue, respectively. In cells transfected with Pirh2 or SR $\beta$ alone, SR $\beta$ was localized in the ER (Figs. 1C, I, and L), whereas Pirh2 was distributed throughout both the nucleus and cytoplasm (Figs. 1G, J, and M). When both Pirh2 and SR $\beta$ were transfected into the same cell, Pirh2 was colocalized with SR $\beta$ in the ER (Figs. 1E, H, K, and $\mathrm{N})$. These observations suggest that Pirh2 colocalizes with $\operatorname{SR} \beta$ in the ER.

\section{Pirh2 ubiquitylates SR $\beta$ in vivo as well as in vitro} To examine whether Pirh2 can promote ubiquitylation of SR $\beta$ in vivo, $2 \times$ FLAG-SR $\beta$ and HA-ubiquitin expression plasmids were cotransfected into HEK293 cells together with Xpress-Pirh2 or -Pirh2 RING mutant (in which residue cysteine 148 within the RING finger domain was replaced with alanine (C148A)) expression plasmid. The cell extracts were subjected to immunoblotting with anti-FLAG antibody. When HA-tagged ubiquitin was cotransfected together with SR $\beta$, the amount of ubiquitylated SR $\beta$ protein was increased (Fig. 2A, left panel). Cotransfection with wild-type Pirh2 (wt) enhanced ubiquitylation of SR $\beta$, which was not observed in the absence of Pirh2 or when cotransfected with the RING mutant (mt) Pirh2. To demonstrate that the high-molecular-mass species recognized by the antiFLAG antibody is polyubiquitin-conjugated SR $\beta$, SR $\beta$ was immunoprecipitated twice as described in legend for Fig. 2A. Then the immunoprecipitates were subjected to immunoblotting with anti-FLAG antibody for detection of SR $\beta$ as well as with antiHA antibody for detection of the ubiquitin chain (Fig. 2A, right and middle panels). In this experiment, anti-HA antibody detects only HA-ubiquitylated $\operatorname{SR} \beta$ but not HA-ubiquitylation of the other coprecipitated proteins. Immunoblots with antiFLAG and anti-HA antibodies gave ladders with similar mobility shifts identical to the molecular mass of a ubiquitin chain (note that $(\mathrm{Ub})_{1} \mathrm{SR} \beta$ or (Ub) ${ }_{2} \mathrm{SR} \beta$ equally migrated in all panels of Fig. 2). These results indicated that Pirh2 promotes ubiquitylation of SR $\beta$ in a RING finger domain-dependent manner.

We then investigated whether Pirh2-induced ubiquitylation of SR $\beta$ can be reconstituted with purified components. Pirh2-mediated ubiquitylation of SR $\beta$ 
A
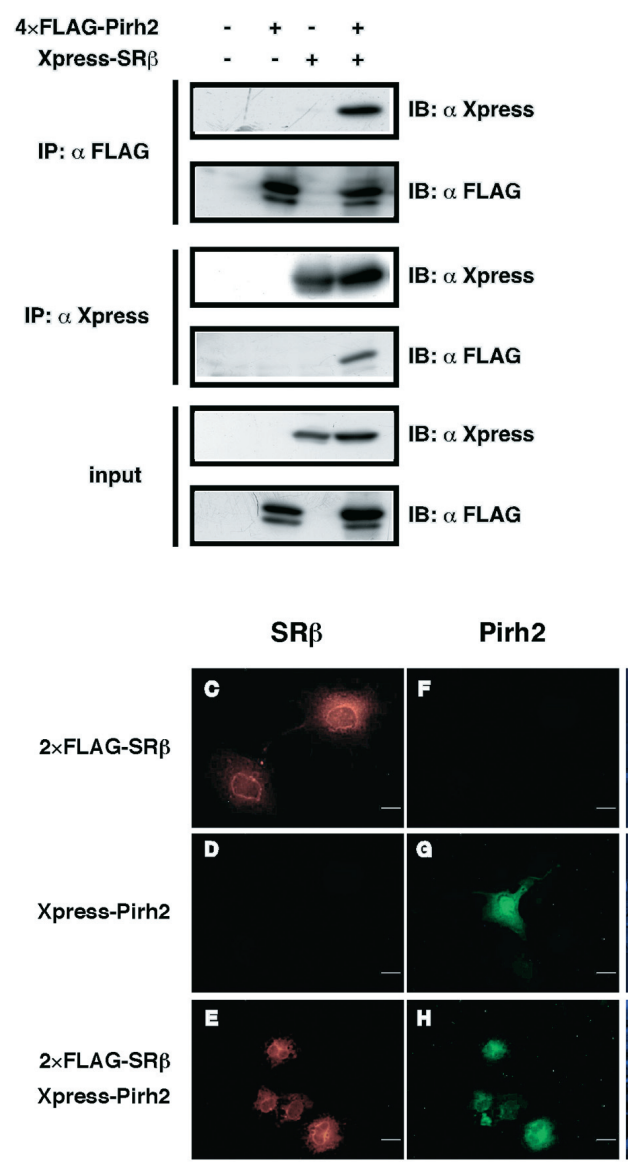

B

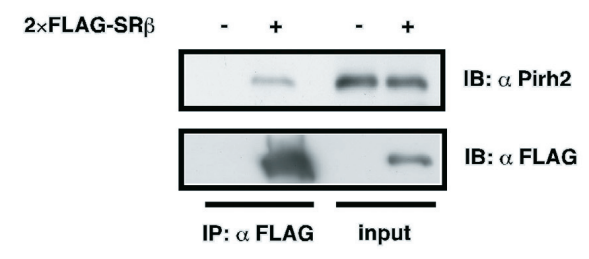

ER
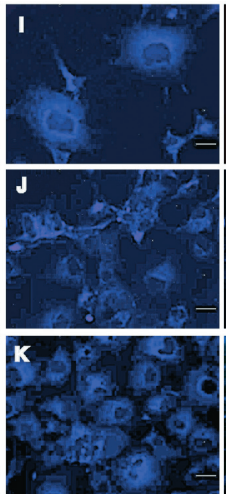

merge
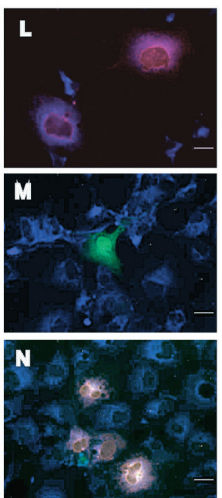

phase contrast

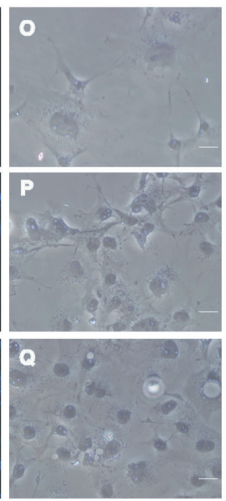

Fig. 1 Pirh2 interacts and colocalizes with SR $\beta$. A: Pirh2 associated with SR $\beta$ in vivo. HEK293 cells were cotransfected with $4 \times$ FLAG-Pirh2 and/or Xpress-SR $\beta$ expression plasmids. After $48 \mathrm{~h}$, the transfected cells were lysed and the cell lysates were subjected to immunoprecipitation (IP) or immunoblotting (IB) with indicated antibodies. B: Exogenous SR $\beta$ interacted with endogenous Pirh2. HEK293 cells were transfected with 2xFLAG-SR $\beta$ expression plasmid and analyzed as described in (A). C-Q: Pirh2 colocalized with SR $\beta$. COS-1 cells were transfected with $2 \times$ FLAG-SR $\beta$ and/or Xpress-Pirh2 expression plasmids. Cells were fixed for immunofluorescence staining with anti-FLAG (red), anti-Xpress (green), and anti-PDI (ER marker, blue) antibodies. Scale bars: $20 \mu \mathrm{m}$.

was detected only when all of the reaction components were present and depended on the existence of an intact RING finger domain of Pirh2 (Fig. 2B). In this in vitro ubiquitylation assay, the high-molecular-mass bands detected by anti-FLAG antibody disappeared when lysine free GST-ubiquitin was employed instead of wild-type GST-ubiquitin (Fig. 2C). This result indicated that SR $\beta$ ubiquitylation induced by Pirh2 directly consisted of a polymerization reaction of the ubiquitin chain but not monoubiquitylation of multiple lysines on SR $\beta$. Therefore, these observations strongly suggested that Pirh2 directly polyubiquitylated SR $\beta$ as a substrate for Pirh2.
Pirh2 is not involved in the regulation of SR $\beta$ protein stability

Polyubiquitin conjugation is a key signal which marks target proteins to the proteasome. We then examined whether Pirh2 can regulate the stability of SR $\beta$ in cells because Pirh2 facilitated the ubiquitylation of SR $\beta$. To investigate the effects of expression of Pirh 2 on SR $\beta$ stability, $2 \times$ FLAG-SR $\beta$ was transiently transfected into U2OS cells with or without Xpress-tagged wild-type Pirh2 or RING finger mutant (C148A) Pirh2. Unexpectedly, the steadystate level of SR $\beta$ was not affected in cells transfected with either wild-type or mutant Pirh2 compared with that in the absence of Pirh2 (Fig. 3A, lanes 1-3). Treatment with proteasome inhibitor, 
A

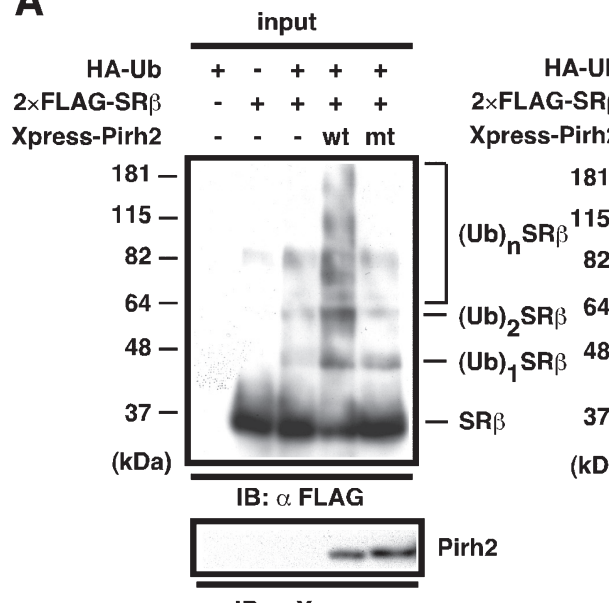

IB: $\alpha$ Xpress

B

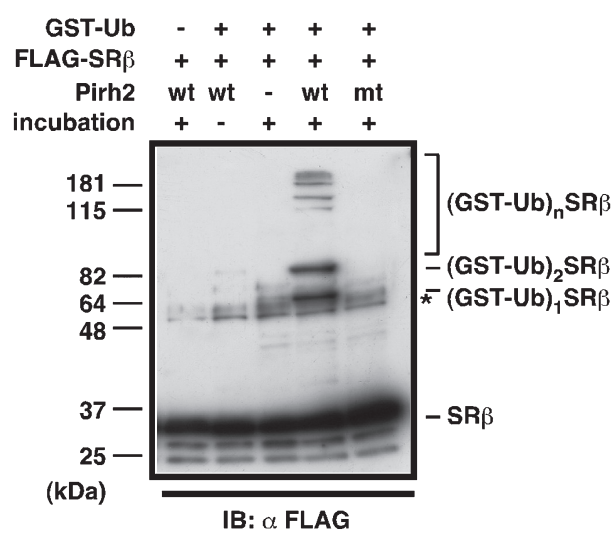

C

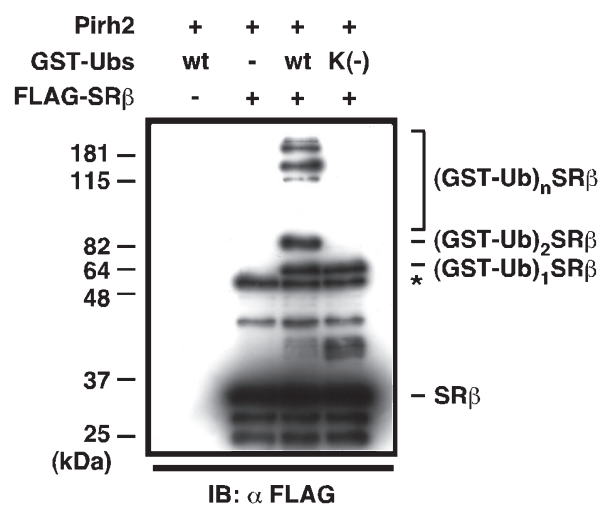

Fig. 2 Pirh2 facilitates ubiquitylation of SR $\beta$. A: Pirh2 mediated ubiquitylation of SR $\beta$ in vivo. HEK293 cells were cotransfected with 2xFLAG-SR $\beta$ and HA-ubiquitin expression plasmids with or without wild-type Pirh2 (wt) or RING finger mutant Pirh2 (mt) expression plasmid. At $40 \mathrm{~h}$ after transfection, cells were treated with $10 \mu \mathrm{M}$ MG132 for $8 \mathrm{~h}$. The cells were lysed, separated by SDS-PAGE, and immunoblotted (IB) with anti-FLAG and anti-Xpress antibodies (input, left panel). Alternatively, the cell lysates were immunoprecipitated (IP) with anti-FLAG antibody. The immunocomplex was denatured in Laemmli's sample buffer containing SDS and 2-mercaptoethanol to dissociate contaminant proteins associated with FLAG-SRB. FLAG$\mathrm{SR} \beta$ was reimmunoprecipitated with anti-FLAG antibody (2xIP: $\alpha \mathrm{FLAG}$ ) and immunoblotted (IB) with anti-HA (middle panel) and anti-FLAG (right panel) antibodies. B: Pirh2 ubiquitylated SR $\beta$ in vitro. Purified FLAG-SR $\beta$ was incubated with GSTubiquitin in the presence or absence of recombinant wild-type Pirh2 (wt) or RING finger mutant Pirh2 (mt) in the in vitro ubiquitylation buffer. The reactants were separated by SDS-PAGE and detected with anti-FLAG antibody. Asterisk shows the bands derived from miss-cleaved GST-tagged FLAG-SR $\beta$. C: Pirh2 induced poly-ubiquitylation but not multi-ubiquitylation of SR $\beta$. Recombinant FLAG-SR $\beta$ was incubated with wild-type GST-ubiquitin (WT) or lysine free GST-ubiquitin (K (-)) together with recombinant Pirh2 in the in vitro ubiquitylation buffer. The reactants were analyzed as described in (B).

MG132 or de novo protein synthesis inhibitor, cycloheximide did not alter the SR $\beta$ expression level regardless of coexpression of Pirh2 or its mutant (Fig. 3A, lanes 4-9). Moreover, we analyzed the effect of Pirh2 expression on the half-life of SR $\beta$ protein by cycloheximide assay. Again, coexpression of wild-type or mutant Pirh2 did not alter the stability of SR $\beta$ protein (Fig. 3B). These data suggested that SR $\beta$ was a stable protein and that Pirh2 did not induce the destabilization of SR $\beta$ protein. To further confirm whether or not Pirh2 regulated the stability of SR $\beta$, we transfected with Pirh2 siRNA to deplete endogenous Pirh2 and assessed the expression level of endogenous SR $\beta$ protein. As shown in Fig. 3C, Pirh2 siRNA but not control siRNA induced a decrease in intracellular Pirh2 in T98G cells. However, ablation of Pirh2 did not alter the expression of SR $\beta$ protein. Taken together, it appeared that Pirh2 did not regulate the amount of SR $\beta$ protein even though Pirh2 promoted ubiquitylation of SR $\beta$. 
A

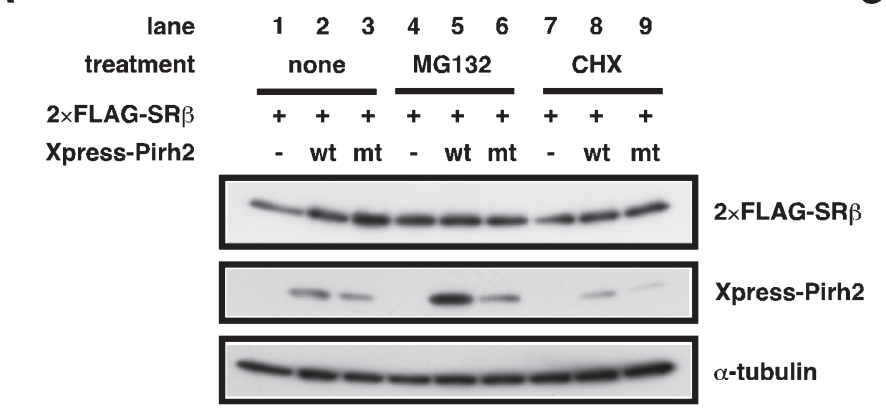

C

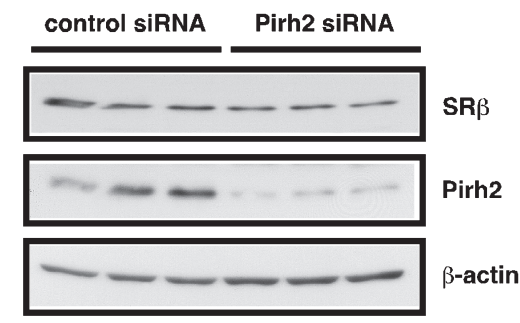

B

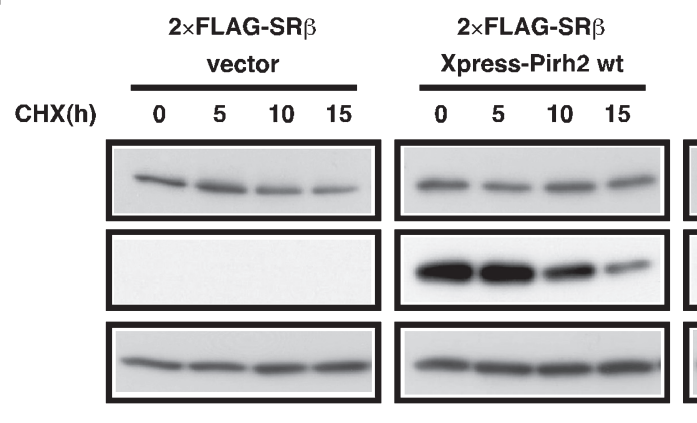

2×FLAG-SR $\beta$ Xpress-Pirh2 mt

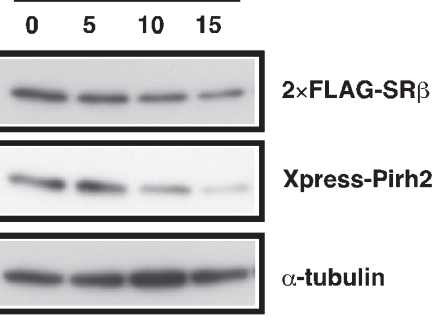

Fig. 3 Pirh2 does not affect the stability of SR $\beta$ protein. A: U2OS cells were cotransfected with $2 \times F L A G-S R \beta$ expression plasmid with or without Xpress-tagged wild-type Pirh2 (wt) or RING finger mutant Pirh2 (mt) expression plasmid. After $36 \mathrm{~h}$, cells were treated with $10 \mu \mathrm{g} / \mathrm{mL}$ cycloheximide $(\mathrm{CHX})$ or $10 \mu \mathrm{M}$ MG132 for $12 \mathrm{~h}$ and then lysed and subjected to immunoblotting with anti-FLAG, anti-Xpress, and anti- $\alpha$-tubulin antibodies. B: To evaluate effects of Pirh2 on the stability of SR $\beta$, cells were transfected with indicated plasmids and harvested at indicated times after cycloheximide treatment. Then, they were lysed and subjected to immunoblotting with anti-FLAG, anti-Xpress, and anti- $a$-tubulin antibodies. C: Ablation of Pirh2 did not alter SR $\beta$ protein expression level. T98G cells were transfected with control siRNA or Pirh2 siRNA in triplicate. The cell lysates were subjected to immunoblotting with antibodies against Pirh2, $\mathrm{SR} \beta$, and $\beta$-actin antibodies.

Pirh2 cannot mediate the polyubiquitylation of SR $\beta$ when lysine residue 6 or 29 of ubiquitin is substituted with arginine

The formation of a polyubiquitin chain by linkage of one ubiquitin moiety to lysine 48 (K48) of the adjacent ubiquitin had been thought to be a signal marking the target proteins for proteolysis by the 26 $\mathrm{S}$ proteasome. However, recent studies demonstrated that polyubiquitin chains can also be assembled via lysine residues of ubiquitin other than K48, and the resulting ubiquitin chains function in indistinct biological processes $(4,7,11,15,17,20,21)$. To determine which lysine residues of ubiquitin are required for polyubiquitylation of SR $\beta$ by Pirh2, we performed an in vitro ubiquitylation assay using $6 \times$ His-tagged ubiquitin mutants in which the lysine residue at position $6,11,29,48$, or 63 was individually replaced with arginine (Fig. 4A). As shown in Fig. 4B, Pirh2 was unable to mediate polyubiquitylation of SR $\beta$ with a ubiquitin mutant K6R and K29R. These data suggested that SR $\beta$ polyubiquitylation mediated by Pirh2 was assembled via lysine residues 6 and/or 29 of the ubiquitin, consistent with the notion that Pirh2 did not regulate SR $\beta$ protein stability.

\section{DISCUSSION}

We reported here for the first time that Pirh2 interacts with and ubiquitylates SR $\beta$ essential for the protein translocation across the ER in the synthesis of secretory protein. Immunocytochemical studies revealed that Pirh2 colocalized with SR $\beta$ in the ER when the two expression plasmids were cotransfected into the same cells. On the other hand, Pirh2 was widely distributed throughout the cells when Pirh2 was transfected alone (Figs.1C-Q). These data suggested that Pirh2 can bind to SR $\beta$ on the ER and may function as a ubiquitin ligase for $\operatorname{SR} \beta$.

Unexpectedly, different from the case of other Pirh2 substrates such as p53, HDAC1 and $27^{\text {Kip } 1}$, neither overexpression nor depletion of Pirh2 affected the expression level and stability of the SR $\beta$ protein. Our studies further suggested that Pirh2 
A

ubiquitin

\begin{tabular}{|c|c|c|c|c|}
\hline wt & K6 K11 & K29 & K48 & K63 \\
\hline K6R & 跑 K11 & K29 & K48 & K63 \\
\hline K11R & K6 R̂́1́t & K29 & K48 & K63 \\
\hline K29R & K6 K11 & R2929 & K48 & K63 \\
\hline K48R & K6 K11 & K29 & R48 & K63 \\
\hline K63R & K6 K11 & K29 & K48 & Rिक \\
\hline
\end{tabular}

B

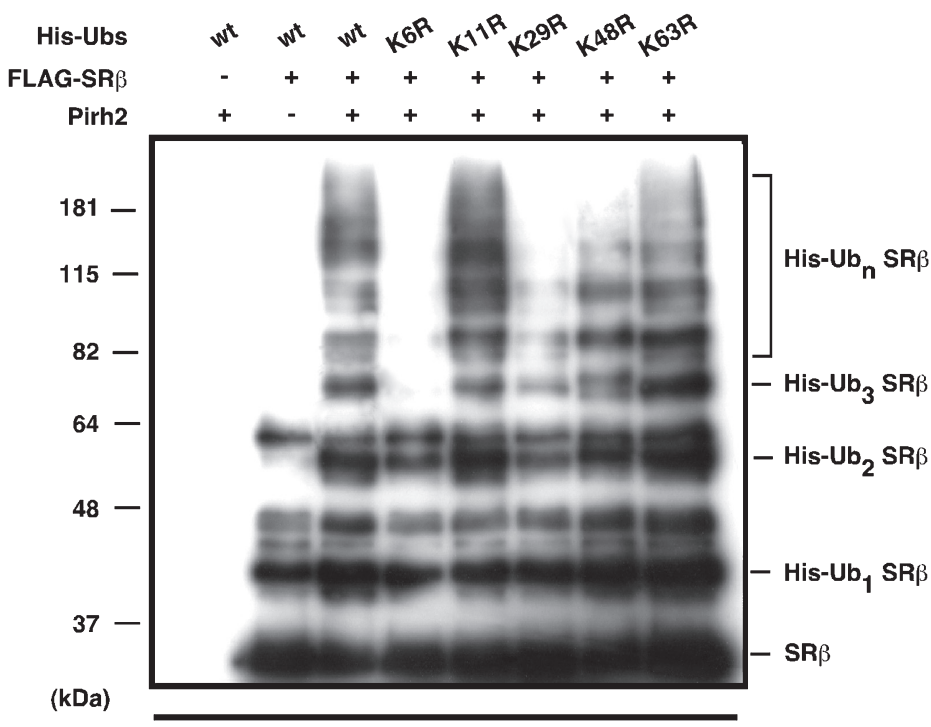

IB: $\alpha$ FLAG

wt K6R K11R K29R K48R K63R

Fig. 4 Pirh2 cannot utilize ubiquitin mutants in which lysine residue 6 or 29 is individually substituted for arginine in mediating the polyubiquitylation of SR $\beta$. A: Schematic representation of His-tagged ubiquitin mutants in which the lysine residues at positions $6,11,29,48$, or 63 were individually substituted for arginine. B: In vitro ubiquitylation assay was performed with recombinant FLAG-SR $\beta$ and Pirh2 in the presence of wild-type ubiquitin (wt) or the ubiquitin mutants shown in (A). The reactants were analyzed as described in Fig. $2 \mathrm{C}$. The amount of each ubiquitin mutant was confirmed by coomassie blue staining (bottom panel).

preferentially utilized lysine residues 6 and 29 of the ubiquitin to mediate the polyubiquitylation of $\mathrm{SR} \beta$. The function of lysine 6-linked polyubiquitylation is yet to be determined. However, given that the lysine 6-linked polyubiquitin chain is not a proteasomal degradation signal, it is suggested that ubiquitylation of SR $\beta$ by Pirh2 does not serve exclusively as the degradation signal for the proteasome. As discussed above, lysine 6-linked polyubiquitylation of SR $\beta$ mediated by Pirh2 may serve as a regulatory ubiquitylation for the SRP-SR complex in the synthesis of secretory proteins without affecting the stability of SR $\beta$ per se. In any case, further studies are required to determine which lysine residues Pirh2 preferentially utilizes in ubiquitylating $\operatorname{SR} \beta$ in vivo.

Recently, it was reported that Pirh2 ubiquitylated $\varepsilon$-COP, a subunit of the COP I coatomer complex, which coats the Golgi-derived vesicles involved in protein transport from the Golgi apparatus to the ER and within the Golgi apparatus $(16,19)$, and consequently promoted the degradation of $\varepsilon$-COP (14).
Overexpression of Pirh2 in a prostate cancer cell line caused downregulation of the secretion of prostate-specific antigen (PSA), a secretory protein in prostate epithelial cells (14). Therefore, Pirh2 may control not only secretion of PSA but also the secretory protein synthesis machinery itself by promoting ubiquitylation of SR $\beta$.

Thus, it is probable that Pirh2 functions not only in cancer cell proliferation by promoting the degradation of $\mathrm{p} 53$, HDAC1 and $\mathrm{p} 27^{k i p l}$ in a ubiquitinproteasome pathway, but functions also in the synthesis of secretory protein by the functional regulation of SR $\beta$ via interaction or ubiquitylation.

\section{Acknowledgements}

We thank Dr. Hirotoshi Kikuchi for helpful discussions and Kazutoshi Asano, Sayuri Suzuki, Tomomi Abe, and Daisuke Yamada for technical assistance. This work was supported in part by Grants-in-aid for Science Research from the Ministry of Education, Science, Sports, Culture and Technology of Ja- 
pan (M.K., K.K., T.H., and C.U.) and COE program of Hamamatsu University School of Medicine funded by the Ministry of Education, Science, Sports, Culture and Technology of Japan (M.K. and K.A.).

\section{REFERENCES}

1. Beitel LK, Elhaji YA, Lumbroso R, Wing SS, Panet-Raymond V, Gottlieb B, Pinky L and Trifiro MA (2002) Cloning and characterization of an androgen receptor N-terminal-interacting protein with ubiquitin-protein ligase activity. $J \mathrm{Mol}$ Endocrinol 29, 41-60.

2. Chau V, Tobias JW, Bachmair A, Marriott D, Ecker DJ, Gonda DK and Varshavsky A (1989) A multiubiquitin chain is confined to specific lysine in a targeted short-lived protein. Science 243, 1576-1583.

3. Chen M, Cortay JC, Logan IR, Sapountzi V, Robson CN and Gerlier D (2005) Inhibition of ubiquitination and stabilization of human ubiquitin E3 ligase PIRH2 by measles virus phosphoprotein. J Virol 79, 11824-11836.

4. Deng L, Wang C, Spencer E, Yang L, Braun A, You J, Staughter C, Pickart C and Chen ZJ (2000) Activation of the I $\kappa$ B kinase complex by TRAF6 requires a dimeric ubiquitinconjugating enzyme complex and a unique polyubiquitin chain. Cell 103, 351-361.

5. Duan S, Yao Z, Hou D, Wu Z, Zhu WG and Wu M (2007) Phosphorylation of Pirh2 by Calmodulin-dependent kinase II impairs its ability to ubiquitinate p53. EMBO J 26, 30623074.

6. Egea PF, Strond RM and Walter P (2005) Targeting proteins to membranes: structure of the signal recognition of particle. Curr Opin Struct Biol 15, 213-220.

7. Galan JM and Tsapis RH (1997) Ubiquitin Lys63 is involved in ubiquitination of a yeast plasma membrane protein. $E M B O$ $J$ 16, 5847-5854.

8. Halic M and Beckmann R (2005) The signal recognition particle and its interactions during protein targeting. Curr Opin Struct Biol 15, 116-125.

9. Hattori T, Isobe T, Abe K, Kikuchi H, Kitagawa K, Oda T, Uchida C and Kitagawa M (2007) Pirh2 promotes ubiquitindependent degradation of the CDK inhibitor p2 $7^{k i p 1}$. Cancer Res 67, 10789-10795.

10. Hershko A and Ciechanover A (1998) The ubiquitin system.
Annu Rev Biochem 67, 425-479.

11. Hofmann RM and Pickart CM (1999) Noncanonical MMS2encoded ubiquitin-conjugating enzyme functions in assembly of novel polyubiquitin chains for DNA repair. Cell 96, 645653.

12. Leng RP, Lin $\mathrm{Y}, \mathrm{Ma} \mathrm{W}, \mathrm{Wu} \mathrm{H}$, Lemmers B, Chung S, Parant JM, Lozano G, Hakem R and Benchimol S (2003) Pirh2, a p53-induced ubiquitin-protein ligase, promotes p53 degradation. Cell 112, 779-791.

13. Logan IR, Gaughan L, McCracken SRC, Sapountzi V, Leung HY and Robson CN (2006) Human PIRH2 enhances androgen receptor signaling through Inhibition of histone deacetylase 1 and Is overexpressed in prostate cancer. Mol Cell Biol 26, 6502-6510.

14. Maruyama S, Miyajima N, Bohgaki M, Tsukiyama T, Shigemura M, Nonomura K and Hatakeyama S (2008) Ubiquitylation of $\varepsilon$-COP by PIRH2 and regulation of the secretion of PSA. Mol Cell Biochem 307, 73-82.

15. Nishikawa H, Ooka S, Sato K, Arima K, Okamoto J, Klevit RE, Fukuda M and Ohta T (2004) Mass spectrometric and mutational analyses reveal Lys-6-linked polyubiquitin chains catalyzed by BRCA1-BARD1 ubiquitin ligase. $J$ Biol Chem 279, 3916-3924.

16. Ostermann J, Orci L, Tani K, Amherdt M, Ravazzola M, Elazar Z and Rothman JE (1993) Stepwise assembly of functionally active transport vesicles. Cell 75, 1015-1025.

17. Pickart CM (2001) Ubiquitin enters the new millenium. Mol Cell 8, 499-504.

18. Rogan IR, Sapountzi V, Gaughan L, Neal DE and Robson CN (2003) Control of human PIRH2 protein stability: involvement of TIP60 and the proteasome. J Biol Chem 279, 11696-11704.

19. Rothman JE (1994) Mechanisms of intracellular protein transport. Nature 372, 55-63.

20. Spence J, Gail RR, Dittmar G, Sherman F, Karin M and Finley D (2000) Cell cycle-regulated modification of the ribosome by a variant multiubiquitin chain. Cell 102, 67-76.

21. Spence J, Sadis S, Haas AL and Finley D (1995) A ubiquitin mutant with specific defects in DNA repair and multiubiquitination. Mol Cell Biol 15, 1265-1273.

22. Zhang L, Li J, Wang C, Ma Y and Huo K (2005) A new human gene hNTKL-BP1 interacts with hPirh2. Biochem Biophys Res Commun 330, 293-297. 